\title{
POŠTA
}

TELEKOMUNIKACIE A

ELEKTRONICKY OBCHOD

\section{STRATEGISCHES MANAGEMENT IM POSTINDUSTRIELLEN ZEITALTER = WISSENSMANAGEMENT DURCH INFORMATION UND KOMMUNIKATION}

\author{
Rudolf Brabeck ${ }^{1}$ Katarína Štofková ${ }^{2}$
}

\section{Das Dilemma des klassischen Strategischen Managements}

Die permanenten und höchst dynamischen Verschiebungen von Umwelt- und Umfeldbedingungen (Gomez/Probst - 1995 - S. 5 ff. sowie Rosenstiel/Comelli - 1998) erzwingen vor allem von sozio-ökonomischen Systemen eine rasche und enorm flexible Anpassung und strategische Integration dieser gravierenden Veränderungen in das unternehmerische Zielsystem.

Eine nur auszugs- und stichwortartige Übersicht über die beherrschenden Hintergrundfaktoren (,shifts und drifts') des gegenwärtigen Wandels soll folgende Übersicht liefern.

Soziale Megatrends:

- Demographische Probleme (Beispiele: tendenzielle Überalterung und Bevölkerungsrückgang, dies gilt vor allem für fortgeschrittene europäische Volkswirtschaften),

- Substitution traditioneller Werte (Beispiel: der Beruf als Lebensmittelpunkt wird durch die individuelle Konzentration auf die persönliche Entfaltung verdrängt - allgemein ist feststellbar, daß wir eine stetige und massive Verlagerung von idealistischen zu konsumtiven, teilweise immateriellen Werten erleben),

- abnehmende soziale und finanzielle Sicherheiten und zukünftig drohende Verarmung breiter Bevölkerungs- und Erwerbsschichten (die wesentliche Ursache: der sog. Markt wird mehr und mehr zum einzigen grundlegenden Regulierungs- und Ordnungssystem von zahlreichen Lebensbereichen, deshalb erfolgt die verstärkte Anmahnung sozialer Verantwortung und politischer Korrekturen),

- breite gesellschaftliche Wahrnehmung von ökologischen Herausforderungen und interkulturellen Bedrohungen sowie von absehbaren Engpässen bei der Versorgung mit Rohstoffen und Energie.

Wirtschaftliche Megatrends:

- Verstärkung von Globalisierungs- und Internationalisierungstendenzen (vor allem: grenzüberschreitende Logistikketten und intensivste Vernetzung und Outsourcing von

\footnotetext{
${ }^{1}$ Diplom-Kaufmann Rudolf Brabeck, Fakultät für Management und Ökonomie des Transports und der Kommunikation, Universität Žilina, Rudolf@Brabeck.de

${ }^{2}$ Ing. Katarina Štofková, Fakultät für Management und Ökonomie des Transports und der Kommunikation, Universität Žilina, Katarina.Stofkova@fpedas.utc.sk
} 
Unternehmen im Rahmen der effektivsten Allokation von monetären und ProduktionsRessourcen),

- stark sinkende Bedeutung des Sekundärsektors für sog. altindustrialisierte Regionen, aber nicht spiegelbildlich zunehmender Anteil des tertiären Sektors,

- fast weltweite Durchgängigkeit von Käufermärkten,

- Realisierung von Wertschöpfungs- / strukturellen Flexibilisierungsansätzen (Beispiele: Reengineering, Benchmarking, Wertkettenanalysen, Lean Management),

- Marktsättigung auf fast allen klassischen Märkten.

Technologische Megatrends:

- Dominanz von Wissen (durch Information und Kommunikation),

- Revolution in Basistechnologien,

- Integration von Daten- und Nachrichtensystemen,

- Informations- und Kommunikationstechnologien werden schnell zu den dominierenden Schlüsselfaktoren sowohl im gesellschaftlichen Leben als auch in der unternehmerischen Wertschöpfungskette.

Diese uns völlig fremde Unsicherheit - bis dato kannten wir nur das ,fast automatische' Wachsen des Wohlstands als Konstante - prägt einerseits unser Zeitalter der ökonomischen und gesellschaftlichen Diskontinuität. Andererseits überfordern diese Turbulenzen häufig strategische (langfristig ausgerichtete) Orientierungen, nachdem die kurz skizzierten Umwelt- und Umfeldfaktoren (Risikodimensionen) eine immer raschere und flexiblere Anpassung von Unternehmen bedingen.

Welche Rahmenbedingungen und ,tasks' für das ,Management des Wandels im 21. Jahrhundert' sind daraus abzuleiten?

- Gefahr der Kurzfristmentalität managerialen Denkens und Entscheidens (Überlagerung durch aktuell bedeutsame Ereignisse),

- Notwendigkeit der Erhöhung der Reaktionsgeschwindigkeit,

- stetige Aufgabe zur Früherkennung von Megatrends,

- Konzentration auf die Gesamtpositionierung des Unternehmens (keine isolierte Optimierung von einzelnen Funktionsbereichen),

- generelle Pflicht zur Generierung und Absicherung von langfristigen Erfolgspotentialen (Tschirky - $1998-$ S. 216).

Unternehmen, die ihr spezifisches Know-how - also ihre geballte Fach- und ihre Sachkompetenz sowie ihr Wissen - mit ihrer Informations- und Kommunikationstechnologie intelligent bzw. effizient verknüpfen können, werden sich durch das dadurch verfügbare implizite und explizite Wissen ihrer Mitarbeiterschaft dauerhafte strategische Wettbewerbsvorteile verschaffen können. Vor allem ermöglicht das Wissensmanagement, folgende Potentiale zu realisieren:

- Transparenz und Nutzung von internen Wissensvorräten (,wenn Siemens wüsste, was Siemens weiß') und

- Verbesserung der Marktposition (schnellere Entwicklung und Umsetzung von Innovationen, höherer Nutzen und intensivere Bindung der Kunden). 


\section{Grundlagen zum strategischen Konzept des Managements von Wissen}

Jeder strategisch formulierte Managementansatz konzentriert sich auf die nachhaltige Verbesserung der Wettbewerbsfähigkeit und die Analyse sowie die Beeinflussung kritischer Erfolgsfaktoren. Dies ist auch typisch für das Konzept des ,Knowledge Management', das aber den Produktionsfaktor Wissen im Rahmen der Unternehmensführung am stärksten betont.

In diesem Kontext sei angemerkt, daß Wissensmanagement weit über den Begriff Informationsmanagement hinausgeht. Das Informationsmanagement hat ,nur' die Frage zu beantworten: welche Information wird in welcher Form zu welchem Zeitpunkt an welchem Ort benötigt?

Ganz besonderes Augenmerk richtet das Wissensmanagement einerseits darauf, die Felder (1) Technologiemanagement (Infrastruktur) und (2) Management der Organisation (organisationaler Aufbau) zu verbinden.

Andererseits sind (3) das Management der Unternehmenskultur und das (4) Human Resources Management $\mathrm{zu}$ verknüpfen - selbstverständlich müssen grundsätzlich alle Maßnahmen des Wissensmanagements auf jeder qualitativen und jeder quantitativen Ebene der Unternehmensführung ansetzen (Vision, Strukturen und Instrumente wie Intranet oder Datenbanken etc.).

Das Wissensmanagement ist als lateral wirkender Nucleus betrieblicher Veränderungen zu klassifizieren (Hopfenbeck - 2002 - S. 117 ff.). Vor diesem Hintergrund sind vielschichtige Konsequenzen absehbar, insbesondere ist zu erwarten, daß

- abteilungsübergreifende Wissensclusters in Unternehmen entstehen werden (,Wissens-, Projekt- und Macht-Netzwerke'), daraus ergibt sich eine tendenzielle Aushöhlung der traditionellen Hierarchie,

- Mobilität (Tele-Arbeit) und Dezentralisierung in kurzer Zeit erheblich an Bedeutung gewinnen werden und

- Bildung (Aus- und Weiter- sowie Fortbildung bzw. ,life-long-learning') das sowohl individuell als auch gesamtgesellschaftlich wichtigste Kapital verkörpert.

\section{Kernelemente des Wissensmanagements}

Wissensorientiertes Management muß unterschiedliche Module des Wissens formulieren, Wissenslücken aufdecken und zukunftweisende Informations- und Wissensfelder definieren sowie diese Bausteine zu einer Wissens-Strategie integrieren.

\subsection{Was zu wissen ist}

Gebraucht werden detaillierte Informationen über wissensorientierte Ziele sowohl im strategischen als auch im operativen Bereich.

Dazu benötigt wird die volle Unterstützung des gesamten Management-Systems.

Alle Wissensziele werden erfahrungsgemäß durch

- den formalen Austausch in der Hierarchie (,Wissensbedarfs-Gespräche'),

- Bildungsbedarfsanalysen,

- inhaltliche Know-how-Bilanzen und

- Zielvereinbarungsinstrumente der Personalentwicklung

transparent gemacht (Bullinger et al. - $1998-$ S. 12).

\subsection{Wer Wissen trägt}

Je größer und internationaler Unternehmen strukturiert sind, desto umfassender ist die ,Wissens-Pathologie' ausgeprägt (Gründe: Duplizität von Entwicklungen, parallele Dokumentationen etc.). 
Wer verkörpert insbesondere Wissen?

- Stakeholders (z.B.: Kunden, Lieferanten),

- Experten und Suchmaschinen,

- Wissenskarten,

- öffentliche Einrichtungen (z.B.: Universitäten),

- ,think tanks',

- digitale Speicher (z.B.: Abfrage über Algorithmen).

\subsection{Hindernisse bei der Akquisition von Wissen}

Der Rückgriff auf externe Wissensquellen ist die häufigste Form der Aneignung von Wissen.

Aber dieser Wissenserwerb provoziert geradezu interne Barrieren der mangelnden Akzeptanz. Hinzu kommt, daß Wissensmärkte in hohem Maße als intransparent gelten.

\subsection{Wie Wissen entwickelt wird}

Zentrales Ziel dieses Moduls ist der Aufbau der Fähigkeit, Wissen systematisch zu generieren (um neue Produkte, Ideen und Prozesse anbieten zu können).

Die übergeordnete Rolle wird dem Funktionsbereich Organisation zugewiesen, um individuelle Wissensentwicklungsprozesse durch die Initiierung von

- Vorschlagswesen,

- monetären Benefits,

- aber auch von motivationalen Anreizen,

- persönlichen Freiräumen,

- Innovationsmanagement etc.

und kollektive Wissensentwicklungsprozesse durch

- Schaffung von Teams und Arbeits- sowie Projektgruppen,

- Kompetenzzentren,

- simulierte Szenarien und

- Projektauswertungen etc.

,anzuschieben' (Probst et al. - 1998, S. 183 ff.).

\subsection{Wie Wissen transferiert wird}

Jedes erfolgreiche Unternehmen setzt voraus, daß Wissen geteilt werden kann (Geißler -1998 - S. 38 ff.).

Die Steuerung von Wissensverteilung bedingt einerseits eine entsprechende technische Infrastruktur wie

- Datennetze,

- Intranet,

- eMail,

- Management-Informations-Systeme etc.,

andererseits eine adäquate organisatorische Infrastruktur, insbesondere die exakte Definition der Zugangsberechtigung. 
In diesem Zusammenhang treten häufig sog. Teilungsbarrieren auf (die häufigsten Gründe: Wissen veraltet zu schnell, Zeitdruck, individuelle ,Gegen-Interessen', kurzfristige Orientierung etc.).

\section{Voraussetzungen der Implementierung des Wissensmanagements}

Die ursprüngliche Euphorie im Hinblick auf das Wissensmanagement hat zwar dazu geführt, daß von vielen Mitarbeitern zahlreiche Informationen eines Unternehmens sehr intensiv genutzt werden, die jedoch für den betrieblichen Prozeß der Leistungserstellung völlig irrelevant sind.

Der produktivitätssteigernde Input von Wissen scheitert aber in der betrieblichen Realität relativ oft an der Wissensintelligenz, wenn die gegebene Unternehmenskultur die Weitergabe von Wissen nicht förderlich beeinflusst.

Dies ist häufig dann gegeben, wenn der interne Transfer von Wissen aus Angst geblockt wird, weil ,potentielle Konkurrenten' (Kollegen, die das zu transferierende Wissen verwenden würden) daraus nutzbares ,Herrschafts-Wissen' entwickeln und sich somit ganz erhebliche ,Karriere-Vorteile' verschaffen können.

Das Wissensmanagement entpuppt sich somit als ein zentrales Thema der Unternehmenskultur.

\section{Literaturquellen:}

[1] Bullinger, H.-J., et al. : Global Networking - Management vernetzter Dienstleistungen, Fraunhofer Institut für Arbeitswissenschaft und Organisation, Stuttgart 1998

[2] Geißler, J.: Lotus Notes als Werkzeug für das Knowledge Management, Regensburg 1998

[3] Gomez, P./Probst, G.: Die Praxis des ganzheitlichen Denkens. Vernetzt denken Unternehmerisch handeln - Persönlich überzeugen. Bern/Stuttgart/Wien, 1995

[4] Hopfenbeck, W.: Allgemeine Betriebswirtschafts- und Managementlehre, Das Unternehmen im Spannungsfeld zwischen ökonomischen, sozialen und ökologischen Interessen, München 2002

[5] Probst, G., et al.: Wissen managen: wie Manager ihre wertvollste Ressource optimal nutzen, Wiesbaden 1998

[6] Rosenstiel, L./Comelli, G.: Auf dem Weg in die Zukunft: Führung zwischen Stabilität und Wandel, Unternehmer-Forum 1998, HypoVereinsbank, München 1998

[7] Tschirky, H.: Konzept und Aufgaben des Integrierten Technologie-Managements, in: Tschirky/Koruna (Hrsg.), Technologie-Management: Idee und Praxis, Zürich 1998

[8] Štofková J., Štofková K.: On the problem of quality management systems and models of quality in services, in: Quality Management of Services. Žilinská univerzita v Žiline, F PEDaS, EDIS 2006. ISBN 80-8070-573-9, s.121-129

[9] Štofková J.: Strategický manažment ako faktor zvyšovania efektívnosti podniku, in: Podniková ekonomika a manžment. Elektronický časopis roč. II, Žilina: ŽU F PEDaS, 1/2006, ISSN 1336-5878, s. 41-45

\section{Grantová podpora:}

- Nem/SR/ŽU 3/07 "Aplikácia cyklu PDCA v službách ako faktor zvyšovania efektívnosti riadenia kvality"

- Pol'/SR/ŽU 1/07 - "Moderné trendy strategického manažmentu a ich aplikácia v podniku ako faktor zvyšovania konkurencieschopnosti". 